\title{
The use of cyclic dipeptide producing LAB with potent anti-aflatoxigenic capability to improve techno-functional properties of clean-label bread
}

\author{
Maryam Ebrahimi ${ }^{1}$, Alireza Sadeghi ${ }^{2^{*}}$ and Seyed Ali Mortazavi ${ }^{3}$
}

\begin{abstract}
Purpose: The aim of the present study was to evaluate the antifungal activity, anti-aflatoxigenic capability, and technological functionality of the selected lactic acid bacteria (LAB) isolated from wheat sourdough.

Methods: The preselected $L A B$ isolates were screened based on their antifungal activities and acidification capacities. Then, the antifungal compounds were identified using gas chromatography/mass spectrometry in the selected LAB culture filtrate obtained from its preparative thin-layer chromatography. The HPLC-based analysis was also used to investigate the anti-aflatoxigenic potentials of the selected $L A B$ isolate. Finally, controlled sourdough (containing selected $L A B$ isolate as starter culture) was used to produce loaf bread, and properties of the product were evaluated in terms of hardness, phytic acid content, overall acceptability, and surface moldiness.

Results: Molecular approaches led to the identification of Pediococcus pentosaceus as the selected LAB isolate. In vitro and in situ antifungal activities of the selected $L A B$ against Aspergillus niger were verified. Antifungal metabolites of the LAB included fatty acid ester, hydroxylated fatty acid ester, an antimicrobial compound, and cyclic dipeptide. Potent anti-aflatoxigenic capabilities of the $L A B$ isolate and cyclic dipeptide (which was identified in the antifungal fraction of the $L A B$ ) were also verified. To our best knowledge, the cyclic dipeptide detected in the present study has never been shown before to possess anti-aflatoxigenic effect. Furthermore, the results revealed that controlled sourdough improved the techno-functional properties of the produced loaf wheat bread.

Conclusion: Altogether, our findings indicate that the selected P. pentosaceus isolate exhibiting proper characteristics can be used as a bio-preservative and bio-improver in the processing of clean-label breads.
\end{abstract}

Keywords: Bio-preservative, Bio-improver, Antifungal metabolites, Anti-aflatoxigenic, Cyclic dipeptide

\section{Introduction}

Fermentation is the best method to improve nutritional, sensorial, and functional properties of cereals. Characterization of multifunctional potentials of lactic acid bacteria (LAB) isolated from cereal-based fermented ecosystems such as sourdough is also pivotal to prepare new starter cultures for bakery industries. Sourdough is a very complex and stressful

\footnotetext{
* Correspondence: sadeghi.gau@gmail.com

${ }^{2}$ Department of Food Science and Technology, Gorgan University of

Agricultural Sciences and Natural Resources, Gorgan 49138-15739, Iran Full list of author information is available at the end of the article
}

niche with numerous potentials to use as a proper alternative for synthetic bakery preservatives and improvers. This ecosystem consists of a mixture of cereal or cereal ingredients and water, fermented with $\mathrm{LAB}$ and yeasts. The sourdough capabilities are mainly related to the metabolism of its predominant $\mathrm{LAB}$. The combination of technological and functional potentials is also necessary to select proper LAB to use as starter, protective, or adjunct cultures in bread making (Gobbetti et al. 2014).

Bread shelf life and quality are limited due to staling and microbial spoilage. Hence, bakery industries are

(c) The Author(s). 2020 Open Access This article is licensed under a Creative Commons Attribution 4.0 International License, which permits use, sharing, adaptation, distribution and reproduction in any medium or format, as long as you give appropriate credit to the original author(s) and the source, provide a link to the Creative Commons licence, and indicate if changes were made. The images or other third party material in this article are included in the article's Creative Commons licence, unless indicated otherwise in a credit line to the material. If material is not included in the article's Creative Commons licence and your intended use is not permitted by statutory regulation or exceeds the permitted use, you will need to obtain permission directly from the copyright holder. To view a copy of this licence, visit http://creativecommons.org/licenses/by/4.0/ 
seeking to find a proper solution to overcome these problems. One of the best ingredients to delay bread staling and prevent its microbial deterioration is controlled sourdough produced with selected LAB as starter cultures. Furthermore, the controlled sourdough has several benefits such as improving shelf life and sensorial, functional, and nutritional properties of the product. This fermented ecosystem can be used as a food-grade biological supplement, bio-preservative agent, and suitable alternative for chemical bakery additives (Clarke and Arendt 2005).

In vitro, in vivo, and in situ antifungal, as well as antiaflatoxigenic potentials of the sourdough LAB have been demonstrated. Synthesis of a wide variety of antagonistic compounds along with their complex synergistic interactions and different competition phenomena of the live cells are involved in the antifungal activity of the LAB (Magnusson and Schnürer 2001; Russo et al. 2017). Some of the most important active ingredients and mechanisms of these inhibitory effects were also revealed (Dalié et al. 2010; Le Lay et al. 2016).

Lactobacillus plantarum which was screened for its in vitro inhibitory activity against spoilage molds was used as sourdough starter culture in Dal Bello et al. (2007) study. Lactic acid, phenyllactic acid, and cyclic dipeptides were also isolated and identified as the major antimicrobial compounds of the Lact. plantarum cellfree supernatant (CFS). Hassan and Bullerman (2008) reported antifungal effect of Lactobacillus paracasei (isolated from a traditional sourdough bread culture) on $\mathrm{Fu}$ sarium, whereas fungal growth was not inhibited by the LAB supernatant. In Garofalo et al. (2012) survey, mold growth was prevented by the most promising antifungal strains (Lactobacillus rossiae and Lactobacillus paralimentarius) on produced sourdough bread. Zhang et al. (2010) demonstrated that the use of sourdough prepared from co-fermentation of Lactobacillus buchneri and Lactobacillus diolivorans delayed growth of indicator molds on the produced bread. It was proposed that cooperative metabolism of these LAB strains led to increased propionate and acetate compared to traditional sourdough.

Among the strains evaluated in Gerez et al. (2013) study, only Lactobacillus fermentum CRL 251 produced thermostable fungi inhibitory peptide. The studies carried out by Le Lay et al. (2016) demonstrated that the screened LAB had proper inhibitory activities against spoilage molds of bakery products. These LAB strains have been introduced as bio-protective cultures with higher antifungal effect than the chemical preservatives. Some technological and functional potentials of predominant LAB starters isolated from sourdough ecosystems such as their antifungal and phytate-degrading activities were also verified (Manini et al. 2016). Hence, this study aims to investigate the antifungal and anti-aflatoxigenic capabilities, as well as techno-functional properties of the selected LAB isolated from wheat sourdough.

\section{Materials and methods}

\section{Raw materials}

White wheat flour (68\% extraction rate) containing $10 \%$ protein, $0.45 \%$ ash content, and $14.20 \%$ moisture content in accordance with AACC (2010) was purchased from a local milling factory. Lact. plantarum (ATCC 1058) as positive control for PCR assay and Aspergillus flavus (ATCC 200026) and Aspergillus niger (ATCC 9029) as indicator aflatoxigenic fungi were purchased from American type culture collection. Active dry yeast extract containing Saccharomyces cerevisiae and microbial media used in the present study were also purchased from a local market and Merck (Germany) company, respectively.

\section{Spontaneous fermentation of wheat sourdough}

For spontaneous fermentation, a mixture of white wheat flour and mineralized water with dough yield (DY = dough weight $\times 100$ /flour weight) of 160 was prepared. On the first day, the mixture was incubated at $37^{\circ} \mathrm{C}$ for $24 \mathrm{~h}$. On the second day, $25 \%$ of the previous fermented sourdough was added to the formulation of new sourdough (back-slopping) and this process was repeated until the final pH would become 4.0 (Rizzello et al. 2010).

\section{Screening strategy}

In the present study, a multi-strategic approach was used to screen predominant LAB isolated from wheat sourdough. Firstly, back-slopping process which leads to maintenance of the most adaptable LAB was applied. Then, surface plating of serially ten-fold diluted mature spontaneous sourdough (final back-slopping) led to isolate the LAB with higher numbers (the most frequent) among the abundant LAB. The spread culture was carried out on deMan Rogosa and Sharpe (MRS) and modified MRS (mMRS; MRS containing 0.05\% L-cysteine/ $\mathrm{HCl}$ and $0.002 \%$ bromophenol blue with final $\mathrm{pH} 6.5 \pm$ 0.2 according to Lee and Lee 2008) agar under microaerophilic conditions $\left(37^{\circ} \mathrm{C}, 24 \mathrm{~h}\right)$. The pure single colonies of streak plate of the LAB isolates were also tested by Gram-staining and catalase assay. Finally, the preselected LAB isolates were subjected to overlay bioassay and in situ acidification analysis.

In vitro antifungal activity of the preselected $L A B$ isolates The overlay method was used to assess the in vitro antifungal activity of the preselected LAB isolates in accordance with Magnusson and Schnürer (2001). After inoculation of the LAB isolates as two 3-cm-long lines on MRS agar and $72-\mathrm{h}$ incubation at $37{ }^{\circ} \mathrm{C}$, the plates 
were overlaid with potato dextrose agar (PDA) containing $10^{4}$ spores $/ \mathrm{mL}$ of $A$. niger or A. flavus. Then, the plates were incubated at $25{ }^{\circ} \mathrm{C}$ and checked by 24 -h intervals in comparison with the control plate.

\section{In situ acidification analysis of the preselected LAB isolates}

Acidification capacity of the preselected LAB isolates was also determined in accordance with the Alfonzo et al. (2013) method. Accordingly, $\Delta \mathrm{pH}$ values (difference between $\mathrm{pH}$ of the incubated control dough and inoculated dough with each preselected LAB isolate) were used to select potentially sourdough starter culture among the preselected LAB isolates. Subsequently, the acidification data were modeled in agreement with the modified Gompertz equation (Zwietering et al. 1990) as follows: $y=k+A \exp \left\{-\exp \left[\left(V_{\max } e / A\right)(\lambda-t)+1\right]\right\}$, where $y$ is the acidification rate at the time $t, k$ is the initial level of the dependent variable to be modeled, $A$ is the $\mathrm{pH}$ variation between inoculation and the initial $\mathrm{pH}$ value, $V_{\max }$ is the maximum acidification rate, and $\lambda$ is the length of the lag phase, respectively.

\section{Molecular identification of the preselected $L A B$ isolates}

Total DNA of the pure single colonies of the selected and non-selected LAB isolates was extracted (DNA extraction kit, Bioneer, AccuPrep K-3032, South Korea), and then, it was subjected to a PCR with F44 (5'GAACGCGAAGAACCTTAC-3') and R1543 (5'GCGTGTGTACAAGACCC-3') primers to amplify 1500 base pairs (bp) target sequence from $16 S$ rDNA in LAB. The amount of PCR reagents (Robust, French) and thermal cycling (Corbett CG1-96, Australia) were also carried out in accordance with Abnous et al. (2009). After agarose gel electrophoresis, the PCR products were sequenced (MWG, Germany). Consequently, the sequencing results were compared with the genomic data available in the national center for biotechnology information (NCBI) using basic local alignment search tool (BLASTn) algorithm (http://blast.ncbi.nlm.nih.gov/Blast). Finally, to investigate the evolutionary relationship between the selected LAB isolate with some LAB isolated from fermented cereals, MEGA 6 software was used. $16 \mathrm{~S}$ rRNA sequence data (Fig. 2) were obtained from the gene bank, and then, they were used to construct a consensus neighbor-joining tree from 1000 bootstrapping replicates (Tamura et al. 2013).

\section{Identification of antifungal compounds in the selected LAB culture filtrate}

Bioactive compounds in antifungal fraction obtained from preparative thin-layer chromatography (TLC: silica gel plates, Merck, Kieselgel, Germany) of the selected LAB culture filtrate were identified using gas chromatography/mass spectrometry (GC/MS: Agilent 7890B, with a nonpolar capillary column- Agilent Hp5Ms, 30-m length, 0.25-mm inner diameter, and $0.25-\mu \mathrm{m}$ film thickness, USA) analysis based on the method described by Lavermicocca et al. (2000). To prepare LAB culture filtrate, overnight culture of the activated $\mathrm{LAB}$ was subjected to cold centrifugation (Hanil, Combi 514R, South Korea; 9000 g, $10 \mathrm{~min}$ ) and microfiltration $(0.22 \mu \mathrm{m})$, respectively. The antifungal fraction of the TLC extract was also determined using inhibitory assay in accordance with Saladino et al. (2016).

\section{Anti-aflatoxigenic capabilities of the selected LAB isolate}

The effect of the selected LAB isolate on aflatoxins $B_{1}$, $B_{2}, G_{1}$, and $G_{2}$ (Sigma-Aldrich, USA) was evaluated in accordance with the HPLC (Waters 2475 HPLC, Multi $\lambda$ Florescence Detector, Marshall Scientific, USA) based method validated in Haskard et al. (2001) research. The inhibitory effects of inactivated LAB cells (autoclaved at $121{ }^{\circ} \mathrm{C}$ for $15 \mathrm{~min}$ ) and the pure metabolites, which were identified in the antifungal fraction (purchased from ALB Technology and Sigma-Aldrich, USA), were also investigated to address the mechanism of the antiaflatoxigenic capability of the LAB isolate using the same method. The concentration of each metabolite, which inhibited by $90 \%\left(\mathrm{IC}_{90}\right)$ of $A$. niger growth, was used for anti-aflatoxigenic assay.

\section{Controlled sourdough}

Pure single colonies of the selected LAB isolate were picked from the agar plates and cultured in MRS broth at $37{ }^{\circ} \mathrm{C}$ for $24 \mathrm{~h}$ to $10^{8}$ colony forming units (CFU). Subsequently, the cells were harvested by cold centrifugation at $5000 \mathrm{~g}$ for $15 \mathrm{~min}$, and then, they were resuspended in sterile water. Next step, the selected LAB isolate was inoculated at $10^{8} \mathrm{CFU} / \mathrm{g}$ of dough to prepare controlled sourdough (DY of 160). Finally, the mixture was fermented at $37{ }^{\circ} \mathrm{C}$ for $24 \mathrm{~h}$ (Dal Bello et al. 2007).

\section{Bread making with the selected LAB isolate}

The formulation of control bread per 100-g flour included 1.2-g active dry yeast extract and 1.5-g NaCl. In order to prepare bread dough, the appropriate amounts of wheat flour and water (60\% absorption) were mixed using a laboratory dough mixer, and then, the dough was left for initial proofing at $35 \pm 1{ }^{\circ} \mathrm{C}$ and $70 \%$ relative humidity for $20 \mathrm{~min}$. After that, the rounded dough pieces were proofed for another $25 \mathrm{~min}$ (final proofing) and subsequently baked in an electrical oven (Feller, Germany) at $220 \pm 5{ }^{\circ} \mathrm{C}$ for $15 \mathrm{~min}$. The produced bread was cooled down to room temperature $\left(25^{\circ} \mathrm{C}\right)$ for $2 \mathrm{~h}$ in the aseptic conditions. To prepare bread samples, $20 \%$ (w/w of flour) of controlled sourdough (containing the selected LAB isolate as starter culture) and spontaneous 
sourdough (containing mature spontaneous sourdough, without starter culture) were added to the dough prepared for the control sample (without sourdough) before the proofing steps, and then, they were processed in the identical conditions (Dal Bello et al. 2007).

\section{Sourdough bread analysis Crumb hardness and porosity}

The texture profile analysis (TPA) was done in order to investigate the crumb hardness using texture analyzer equipment (TAXT, Plus Stable Micro Systems, England) with a cylindrical aluminum probe (25-mm diameter). Briefly, a crosshead speed of $30 \mathrm{~mm} / \mathrm{min}$ was used to compress the crumb samples to $50 \%$ of their original height. Measurements were carried out on slices $(20 \pm 2$ $\mathrm{mm}$ thickness) taken from the center of the loaf, $2 \mathrm{~h}$ after baking (Rizzello et al. 2010). A cross-sectional picture was also scanned (Scanjet G2710 HP, China) from a quarter way through the loaf at the same moment. In order to determine the crumb porosity of samples, the images were converted to a binary form. Afterwards, the image analysis was performed using Image J portable software (version $1.42 \mathrm{e})$ to calculate both the average pore size and the percent area of the pores (Carson et al. 2000).

\section{Phytic acid (PA) content}

Bread samples were extracted with $0.2 \mathrm{~N} \mathrm{HCl}$ (bread concentration $0.1 \mathrm{~g} / \mathrm{mL}$ ), and then, $1 \mathrm{~mL}$ of ammonium iron III solution (Sigma-Aldrich, USA) was added to 0.5 $\mathrm{mL}$ of this extract. Subsequently, the mixture was heated in boiling water bath for $30 \mathrm{~min}$, and consequently, it was cooled in ice water to the same temperature as room. Next step, the contents of tubes was centrifuged at $3000 \mathrm{~g}$ for $30 \mathrm{~min}$. After that, $1 \mathrm{~mL}$ of the supernatant was added to $1.5 \mathrm{~mL}$ of 2, 2'-bipyridine solution (SigmaAldrich, USA). Finally, the absorbance was measured using a spectrophotometer (PG Instruments, LTD T80, England) at $519 \mathrm{~nm}$. The amount of PA was also calculated using a standard curve calibrated with sodium salt of PA (Sigma-Aldrich, USA) as the reference solution (Haug and Lantzsch 1983).

\section{Overall acceptability (OA)}

The 5 -point hedonic scale $(1=$ dislike extremely, $3=$ neither like nor dislike, $5=$ like extremely) was used to evaluate the OA of bread samples, $2 \mathrm{~h}$ after baking. The samples were identified with random codes and evaluated by 12 trained panelists (they had access to the control sample and drinking water, to minimize any error during the test). The bread sensory attributes included the form and shape, chew-ability, color, flavor, and taste. OA was calculated based on the average of the panelist scores (Rizzello et al. 2010).

\section{In situ antifungal activity}

In situ antifungal activity of the selected LAB isolate in bread produced with controlled sourdough was determined through a method described by Gerez et al. (2009) in comparison with the other samples (spontaneous sourdough bread and control wheat bread). In brief, bread samples were inoculated with $3 \mu \mathrm{l}$ of $A$. niger spores $\left(10^{5}\right.$ spore $\left./ \mathrm{g}\right)$ to a blank sterile disc $(6 \mathrm{~mm})$ that was placed at the center of each sample (surface of bread slices). Mold growth was monitored at $25^{\circ} \mathrm{C}$ after 7 storage days through determining the mycelial extension (mold growth area) by Image J software.

\section{Statistical analysis}

All the experiments, as well as the baking trials, were performed three times, and the results were presented as mean values \pm standard deviation. Data were compared by both the one-way analysis of variance and the least significant difference (LSD) post hoc at $P<0.05$ statistical significance with the SPSS-20 software.

\section{Results and discussion Identification of the preselected LAB isolates}

After five-time repetitive back-slopping process, $\mathrm{pH}$ of the sourdough was fixed at value around 4.0. Firstly, several colonies (more than 60 colonies) of predominant $\mathrm{LAB}$ were isolated, and then, four different LAB isolates (Gram-positive, catalase-negative cocci or bacilli) were purified from surface plate of the mature spontaneous sourdough as preselected isolates. There are several reports indicating that sourdough fermentation parameters like back-slopping process can be used successfully to isolate the predominant sourdough LAB. Continuous reinoculation of the prevailing microbiota through backslopping process allows the predominance of some species in sourdough. The number of propagation steps and the frequency of the refreshment times have noticeable impact on the growth of sourdough LAB and their metabolic kinetics due to acidification and nutrient consumption (De Vuyst et al. 2014).

Next step, one of the preselected LAB isolates, which had the highest antifungal activity and the highest acidification capacity, was selected. According to the results of in vitro antifungal assay, isolate 1 had the highest inhibitory activity against indicator fungi in comparison with the other preselected isolates (Fig. 1). Furthermore, inhibitory effect of this LAB isolate on $A$. niger was significantly $(P<0.05)$ higher than its antifungal effect on $A$. flavus, and therefore, this indicator fungus was used in the following antifungal assays. Sourdough is a nonaseptic fermentation system, and therefore, the predominant LAB in this ecosystem can overcome unwanted microorganisms due to their proper antimicrobial activities (De Vuyst et al. 2014). The antifungal activity of 


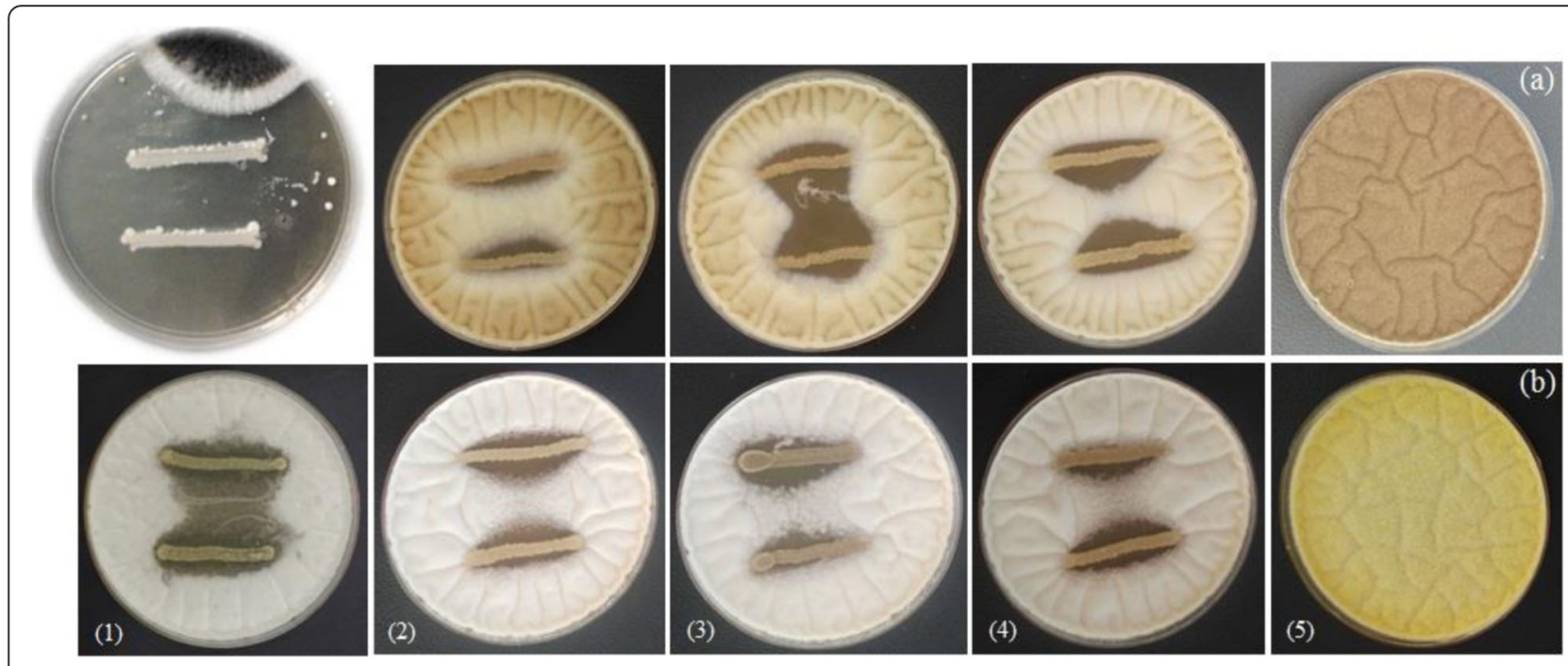

Fig. 1 In vitro inhibitory activity of the preselected LAB isolates in overlay bioassay, against A. niger (the upper pictures) and A. flavus (the lower pictures) in comparison with their controls ( $\mathbf{a}$ and $\mathbf{b}$, respectively). As highlighted, isolate 1 had the highest antifungal activity, and inhibitory effect of the $L A B$ on $A$. niger was significantly $(P<0.05)$ higher than its antifungal effect on $A$. flavus

sourdough $\mathrm{LAB}$, which is attributed to organic acids and the other produced inhibitory metabolites along with their interactions, can affect the complex nature of this antifungal hurdle (Gerez et al. 2009).

The acidification capacity of the LAB isolate 1 was also significantly $(P<0.05)$ higher than the other preselected LAB isolates (Table 1), and therefore, this isolate was used for further characterization as a selected potentially sourdough starter culture. It should be noticed that rapid acidification during sourdough fermentation is an important technological characteristic of LAB. Furthermore, the effect of acidification capacity on several techno-functional attributes of sourdough LAB including antifungal activity, phytate-degrading ability, and dough structure and bread quality characteristics has been verified (Clarke and Arendt 2005).

The results of microscopic and biochemical tests revealed that the selected $\mathrm{LAB}$ isolate was a Gram-positive and catalase-negative cocci. Sequencing results of the PCR products were verified by the BLASTn procedure

Table 1 Acidification kinetics parameters of sourdoughs (dough yield 160) fermented with the preselected $L A B$ isolates (initial cell number $10^{8} \mathrm{CFU} / \mathrm{g}$ ) at $37^{\circ} \mathrm{C}$ for $4 \mathrm{~h}$

\begin{tabular}{llll}
\hline Predominant $L A B$ isolates & $\Delta \mathrm{pH}$ & $V_{\max }(\mathrm{dpH} / \mathrm{h})$ & $\lambda(\mathrm{h})$ \\
\hline Pediococcus pentosaceus & $2.09 \pm 0.07^{\mathrm{a}}$ & $0.79 \pm 0.07^{\mathrm{a}}$ & $0.92 \pm 011^{\mathrm{ab}}$ \\
Pediococcus acidilactici & $1.75 \pm 0.07^{\mathrm{bc}}$ & $0.70 \pm 0.06^{\mathrm{ab}}$ & $0.78 \pm 0.07^{\mathrm{b}}$ \\
Lactobacillus brevis & $1.69 \pm 0.06^{\mathrm{c}}$ & $0.61 \pm 0.06^{\mathrm{b}}$ & $1.05 \pm 0.10^{\mathrm{a}}$ \\
Lactobacillus plantarum & $1.88 \pm 0.04^{\mathrm{b}}$ & $0.69 \pm 0.08^{\mathrm{ab}}$ & $0.85 \pm 0.05^{\mathrm{ab}}$
\end{tabular}

Acidification data were modeled according to the modified Gompertz equation. $\triangle p H$ difference in $\mathrm{pH}$ between the initial value and the value reached after $4 \mathrm{~h}, V_{\max }$ maximum acidification rate, $\lambda$ length of the lag phase. Different letters in the same column indicate a significant difference at $P$ $<0.05$ to confirm the identity of the amplicons. On account of the results, the selected $\mathrm{LAB}$ isolate was identified as Pediococcus pentosaceus. In neighbor-joining dendrogram, the selected isolate was also placed within the Pediococcus group and it was phylogenetically most closely related to Ped. pentosaceus KJ477393.1 (Fig. 2). The other preselected LAB isolates were also identified as Pediococcus acidilactici, Lactobacillus brevis, and Lact. plantarum through the identical procedure. It should be noted that, since the main focus of the present study was on the preselected LAB, and not on the microbial ecology of the sourdough, only the preselected LAB isolates were identified. Microbiological studies have also revealed that LAB group, including Lactobacillus, Pediococcus, Weissella, and Leuconostoc, is more frequent in sourdoughs. It is assumed that the dominance of LAB in sourdoughs is related to their adaptation to this ecosystem and their competitive abilities (De Vuyst et al. 2014).

\section{Antifungal compounds of the selected $L A B$ isolate}

Evaluation of antifungal effect of the TLC spots obtained from Ped. pentosaceus culture filtrate revealed the inhibitory activity of one fraction against $A$. niger (Fig. 3a, b). Five active ingredients including 9,12-octadecadienoic acid (fatty acid), 3-isobutyl 2,5 piperazinedione (antimicrobial compound), 9,12-octadecadienoic acid (Z,Z), 1-methylethyl ester (fatty acid ester), 9-octadecenoic acid (Z), 2-hydroxy1-(hydroxymethyl) ethyl ester (hydroxylated fatty acid ester), and pyrrolo[1,2-a]pyrazine-1,4-dione, hexahydro-7hydroxy-phenylmethyl (cyclic dipeptide) were also detected in the GC/MS chromatogram of this antifungal fraction 


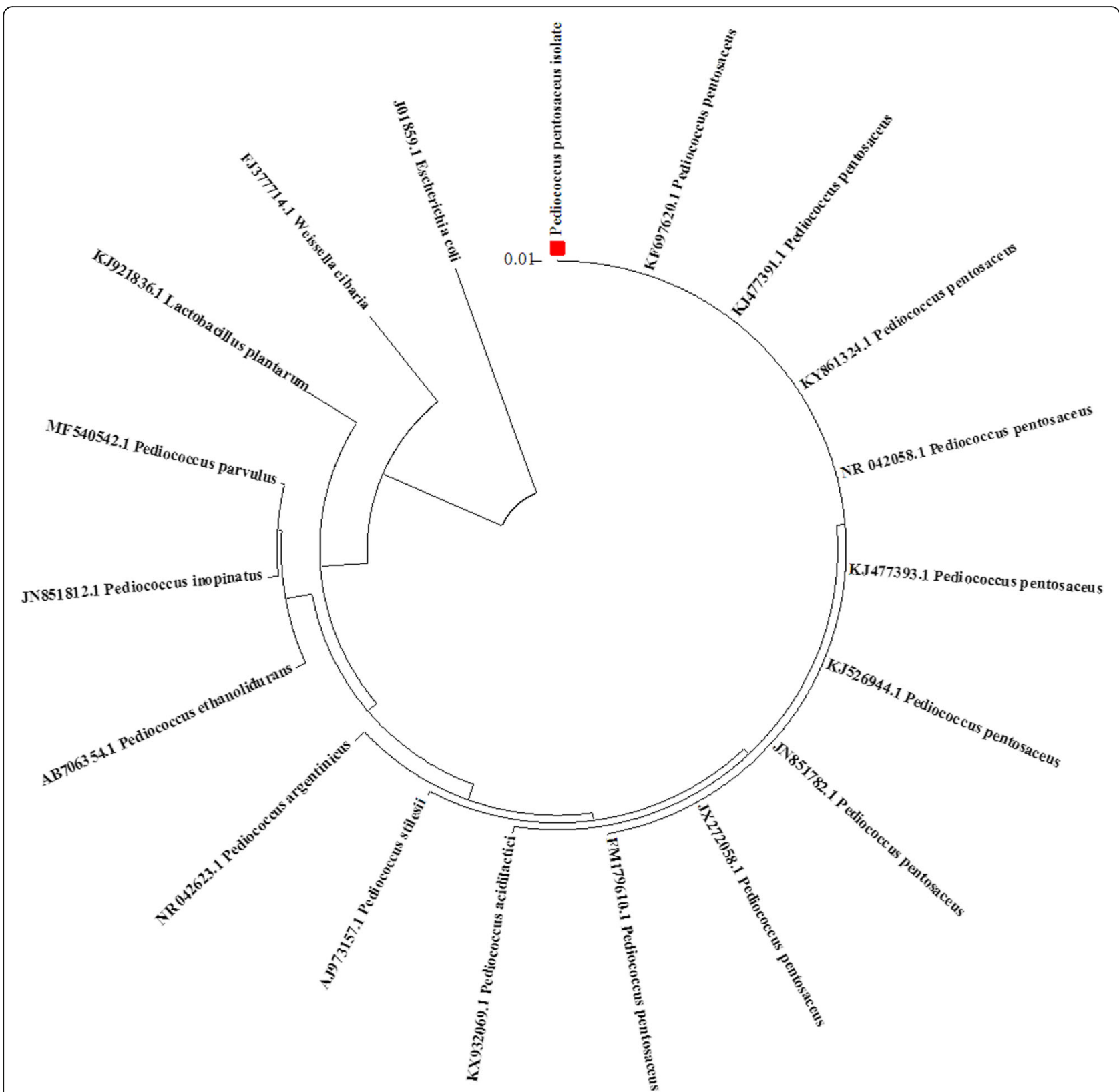

Fig. 2 Phylogenetic tree derived from 165 rRNA gene sequence analysis, giving the position of the selected $L A B$ isolate among the $L A B$ isolated from cereal fermentation. The tree was generated using the neighbor-joining method based on a comparison of approximately $1500 \mathrm{nt}$. Bar indicate $1 \%$ sequence divergence

with retention times of 42.544, 46.066, 47.581, 51.238, and $52.158 \mathrm{~min}$, respectively (Fig. 3c).

The main antifungal compounds produced by LAB include fermentation products such as organic acids, hydroxy fatty acids, proteinaceous substances like antifungal peptides, and low molecular mass inhibitory compounds like cyclic dipeptides (Dalié et al. 2010). The strong in vitro and in situ inhibitory activities of hydroxylated fatty acids and fatty acid esters were reported against a broad spectrum of fungi. This inhibitory effect is related to increase the fungal membrane permeability (Lavermicocca et al. 2000). Furthermore, it is noted that cyclic dipeptides are only active at high concentrations against fungi in comparison with the fatty acid derivatives. Cyclic dipeptides act in synergy with the other inhibitory metabolites produced by LAB, and they are in fact involved in a quorum sensing mechanism for these bacteria (Ström et al. 2002). The presence of phenolic antioxidants and antimicrobial peptides in Sellamani et al. (2016) survey was introduced as responsible for the antifungal activity of 


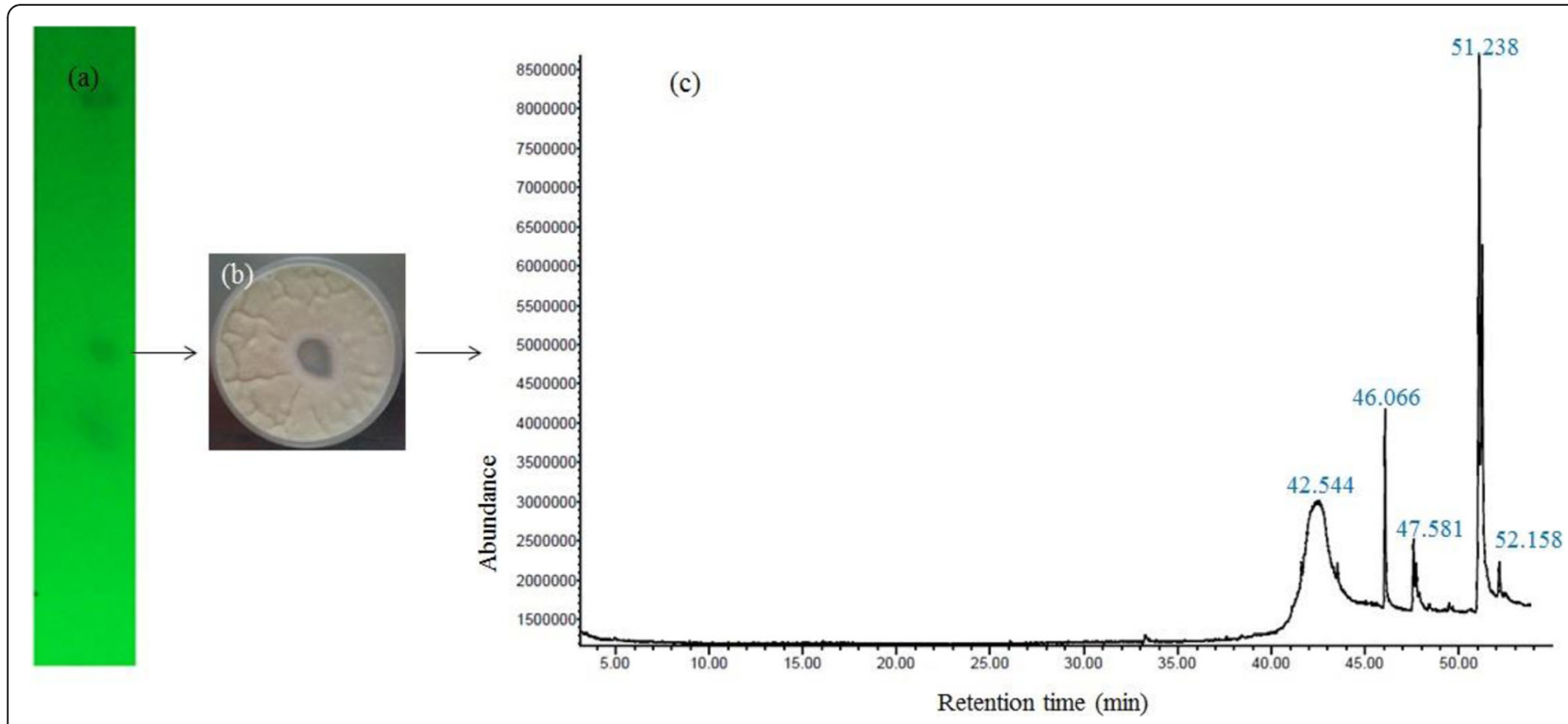

Fig. 3 Preparative thin-layer chromatography (TLC) analysis of the Ped. pentosaceus culture filtrate (a) and the inhibition zone evidenced by the extract of TLC spot, against A. niger (b). Identification of the bioactive compounds of the antifungal fraction obtained from preparative TLC using gas chromatography/mass spectrometry (c)

Ped. pentosaceus isolated from traditional fermented products against Fusarium graminearum. These researchers revealed that the LAB antifungal metabolites led to reduction of the mycelial biomass, change in the structure and function of nuclear DNA, creation of the pores in the mold membrane, inhibition of the ergosterol synthesis, change in the osmotic balance, and induction of the oxidative apoptotic stress.

\section{Anti-aflatoxigenic capability of the selected $L A B$ isolate}

The results obtained from HPLC analysis (Fig. 4) indicated that Ped. pentosaceus isolate reduced $85.50 \pm$ $1.70 \%, 78.27 \pm 1.56 \%, 88.07 \pm 0.74 \%$, and $60.81 \pm 1.16 \%$ of the initial amounts of the aflatoxins $B_{1}, B_{2}, G_{1}$, and $G_{2}$, respectively. Accordingly, the effect of the isolate on aflatoxins $G_{1}$ and $B_{1}$ was significantly $(P<0.05)$ higher than the other toxins. Furthermore, the residue amounts of aflatoxin $\mathrm{B}_{2}$ was significantly lower than the aflatoxin $\mathrm{G}_{2}$. Furthermore, potent anti-aflatoxigenic capability of the inactivated Ped. pentosaceus cells was verified (Fig. 4d), but this inhibitory effect was significantly lower than those of the viable cells $(73.89 \pm 2.06 \%, 75.40 \pm 1.46 \%$, $83.26 \pm 0.74 \%$, and $86.49 \pm 1.16 \%$ reduction of the aflatoxins $B_{1}, B_{2}, G_{1}$, and $G_{2}$, respectively). In accordance with this result, physical absorption plays a considerable role in aflatoxin mitigation by Ped. pentosaceus isolate. Meanwhile, among the pure metabolites, which were identified in the antifungal fraction, only cyclic dipeptide had a noticeable anti-aflatoxigenic effect (Fig. 4e) and the others did not show any inhibitory activity. This cyclic dipeptide $\left(\mathrm{IC}_{90}=35 \mathrm{mg} / \mathrm{ml}\right)$ reduced $74.55 \pm 1.30 \%$,
$79.18 \pm 2.19 \%, 82.06 \pm 1.67 \%$, and $87.32 \pm 2.98 \%$ of the aflatoxins $B_{1}, B_{2}, G_{1}$, and $G_{2}$, respectively.

Due to the mold growth, the presence of mycotoxins in cereals and cereal-based products is a worldwide problem. These toxins are relatively heat stable, and the effect of the baking process on them is also low. Meanwhile, the effect of some sourdough LAB on mycotoxins has been demonstrated. Biological control of aflatoxins by these bacteria revealed that mentioned microorganisms use enzyme-dependent or enzyme-independent reactions to remove or reduce the toxins. The effects of some LAB metabolites like phenyllactic acid, hydroxyphenyllactic acid, and indole lactic acid were also verified on this anti-aflatoxigenic capability (Guimarães et al. 2018). Accordingly, two specific mechanisms including physical binding and inhibition of biosynthesis are involved in the interaction between $\mathrm{LAB}$ and some mycotoxins. Inhibition of aflatoxin biosynthesis by LAB culture filtrate is due to specific bacterial metabolites produced during their growth or cell lysis. Furthermore, the cell wall peptidoglycans of some LAB have been reported to be able to bind some mycotoxins such as aflatoxins through a physical process. Several LAB strains have been found to be able to bind aflatoxins in a fast, reversible, strain- and dose-dependent process (Dalié et al. 2010). Some LAB strains in El-Nezami et al. (1998) study were very effective for trapping aflatoxin $B_{1}$ (more than $80 \%$ ), while aflatoxins $B_{2}, G_{1}$, and $G_{2}$ were less sensitive to this binding process. It is reported that the exposure of more hydrophobic surfaces, peptidoglycan structure, and its amino acid or carbohydrate 
Ebrahimi et al. Annals of Microbiology

(2020) 70:24

Page 8 of 12
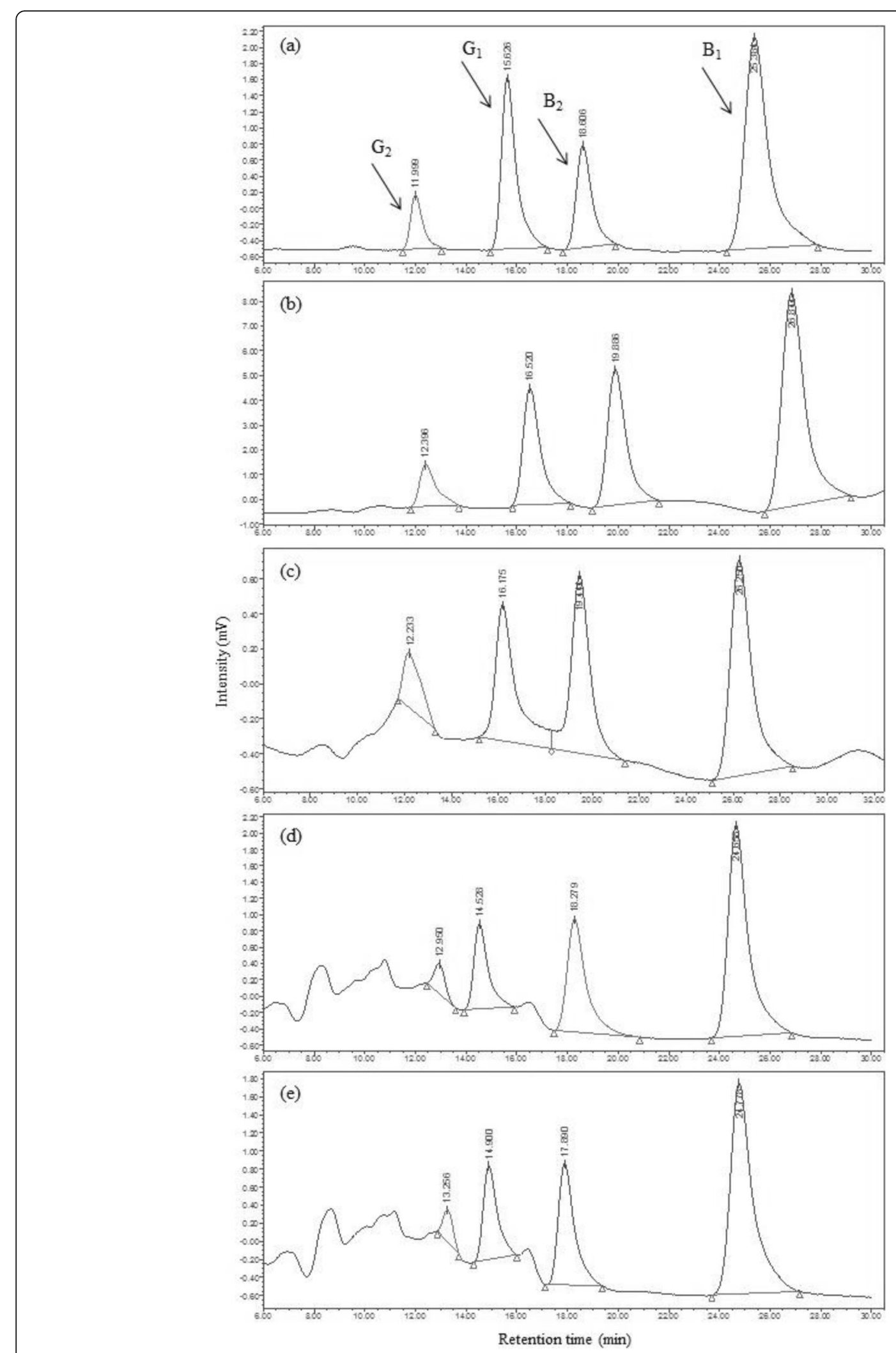

Fig. 4 (See legend on next page.) 
(See figure on previous page.)

Fig. 4 Anti-aflatoxigenic activity of the Ped. pentosaceus isolate. Pure aflatoxins as standard (a); mixture of phosphate-buffered saline (PBS) and aflatoxins as control for matrix effect (b); mixture of Ped. pentosaceus isolate, PBS, and aflatoxins (c); mixture of heat-inactivated Ped. pentosaceus cells, PBS, and aflatoxins (d); and mixture of the pure cyclic dipeptide, which was identified in the antifungal fraction, PBS, and aflatoxins (e) were compared based on the HPLC analysis

composition are involved in this effect (Niderkorn et al. 2009). Mycotoxins are secondary metabolites, and therefore, production of these toxins is correlated to fungal growth. Sellamani et al. (2016) showed that there was a dose-dependent downregulation of zearalenone metabolic pathway genes in treated samples with Ped. pentosaceus culture filtrate. Furthermore, a clear lineage among mycelial biomass, zearalenone gene expression, and its production was reported in this study. Yan et al. (2004) identified a cyclic dipeptide in Achromobacter xylosoxidans CFS as a new inhibitor of aflatoxin that inhibited production of a main precursor of the toxin. Despite the fact that cyclic depeptides have antiaflatoxigenic potentials, the mechanism of this inhibitory effect has not yet been defined. Repression of signal transduction involved in gene expression or direct effect on inhibition of aflatoxin biosynthesis was suggested to explain this capability of cyclic dipeptides. Based on our results, not only physical binding but also other mechanisms are involved in anti-aflatoxigenic effect of Ped. pentosaceus isolate. To our best knowledge, the cyclic dipeptide detected in the present study has never before been shown to possess anti-aflatoxigenic effect.

\section{Sourdough bread properties Crumb hardness and porosity}

As reported in Table 2, crumb hardness of the bread produced with controlled sourdough was significantly $(P<0.05)$ lower than the others, and control bread obviously had the highest hardness. Significant difference in hardness was also observed between bread containing spontaneous sourdough and control sample. Furthermore, crumb porosity in control bread was significantly $(P<0.05)$ lower than the other samples. The porosity of bread containing controlled sourdough was also higher than the bread containing spontaneous sourdough, but there was no significant difference between them (Table 2).
Crumb hardness is a very important characteristic in quality assessment of bread, because of its correlation with bread freshness. The effect of sourdough on reduction of bread firmness is due to improving its volume, regulating the activity of alpha-amylase, and reducing the crystallization of starch. Furthermore, production of microbial metabolites such as proteolytic and amylolytic enzymes, exopolysaccharides, and organic acids during sourdough fermentation has a great influence on this ability. Organic acids play a pivotal role in activation or modification of endogenous enzymes in raw substrates. The interaction of sourdough LAB and yeasts is also another key phenomenon affecting the gas production ability or hydrolysis activities during fermentation (Arendt et al. 2007; Rizzello et al. 2010). Porosity is also a major determiner of the crumb texture affecting the product quality, because it represents retaining of the small bubbles. The increase of porosity in sourdough bread could be attributed to the changes of the gluten network structure caused by enzymatic activity in controlled acidic environment. These phenomena are responsible for better gas-holding capacity. In this condition, gluten network will be unfolded and the exposure of hydrophobic groups will be increased. Consequently, formation of new bonds along with softening effect will improve porosity (Katina et al. 2006). Carbon dioxide in sourdough breads is also produced by both LAB and yeast. Microbial ecology of sourdough, applied baking technology, nutrient supplement of the substrate, and degradation of macromolecules during processing are important in this ability. Meanwhile, the gas retention and not the gas production properties of the dough is responsible for the gas-holding capacity of the sourdough system (Gobbetti 1998).

\section{PA content}

Table 2 shows the PA content of bread samples. As it is shown, the PA content of the bread containing controlled sourdough was remarkably $(P<0.05)$ lower than

Table 2 Properties of the produced loaf breads

\begin{tabular}{|c|c|c|c|c|c|}
\hline \multirow[t]{2}{*}{ Bread samples } & \multicolumn{5}{|c|}{ Bread properties } \\
\hline & Hardness $(N)$ & Porosity (\%) & PA content (mg/100 g) & $\mathrm{OA}$ & A. niger growth (\%) \\
\hline Spontaneous sourdough & $5.89 \pm 0.18^{b}$ & $21.22 \pm 1.08^{a}$ & $192.84 \pm 0.21^{b}$ & $3.94 \pm 0.07^{\mathrm{ab}}$ & $3.89 \pm 0.03^{b}$ \\
\hline Controlled sourdough & $5.02 \pm 0.20^{c}$ & $23.16 \pm 1.58^{a}$ & $180.89 \pm 0.28^{c}$ & $4.06 \pm 0.31^{a}$ & $3.65 \pm 0.28^{b}$ \\
\hline Control & $8.03 \pm 0.12^{\mathrm{a}}$ & $16.65 \pm 0.43^{b}$ & $211.30 \pm 2.135^{a}$ & $3.13 \pm 0.12^{b}$ & $12.37 \pm 2.13^{a}$ \\
\hline
\end{tabular}

Different letters in the same column indicate a significant difference at $P<0.05$. PA phytic acid, OA overall acceptability 
the others. Furthermore, bread sample containing spontaneous sourdough had lower PA content than the control. PA is considered as an anti-nutrient component found in cereals. PA can block the absorption of minerals and trace elements; therefore, a decrease in PA content has functional importance. The effect of controlled sourdough on PA content is attributed to the production of organic acids during sourdough fermentation. It is believed that the low $\mathrm{pH}$ can increase degradation of phytate, and $\mathrm{pH} 4.5-5.0$ was reported as the optimum for this purpose in wheat dough (Leenhardt et al. 2005). In the present study, the $\mathrm{pH}$ of control and bread dough samples containing controlled sourdough and spontaneous sourdough was 5.36, 4.45, and 4.19, respectively. Lopez et al. (2003) reported that prefermentation by LAB increased degradation of phytate up to $90 \%$. As noticed, bread containing controlled sourdough had the most reducing effect on PA content due to its proper $\mathrm{pH}$ and probably phytase activity of the $\mathrm{LAB}$ during the sourdough fermentation. Furthermore, there are evidences showing that some sourdough LAB uses PA as the only carbon source (Palacios et al. 2008).

\section{Bread OA}

From Table 2, it is obvious that the OA of control bread was significantly $(P<0.05)$ lower than the bread containing controlled sourdough. Meanwhile, there was no significant difference in OA between the samples containing controlled and spontaneous sourdoughs. Sensory attributes of bread such as its appearance, oral texture, flavor, and taste have pivotal effect on the consumer's OA. Positive effects of different sourdoughs on sensorial properties of bakery products have been revealed (Katina et al. 2006). It has been demonstrated that the production of flavor precursors (like free amino acids as precursors of isoalcohols), active volatile compounds, and organic acids during sourdough fermentation can change the acceptability of the produced sourdough bread. The influence of sourdough on bread OA is also dependent on the roles of metabolic activities of the microorganisms, endogenous enzymes, fermentation conditions, flour ingredients, and interactions between microbial strains and the substrate ingredients (Clarke and Arendt 2005).

\section{In situ antifungal activity}

As it is shown in Table 2 and Fig. 5, the growth of A. niger on bread containing controlled sourdough and spontaneous sourdough was remarkably $(P<0.05)$ lower than the control sample, after 7 storage days. Fungal spoilage is the most important cause to reduce the quality and shelf life of baked goods, and it leads to economic losses. It is revealed that the mold spoilage of bread was prevented by sourdough LAB as a novel strategy to produce clean-label bread. Control of mold in bakery products by this potent bio-preservation technique is important not only to reduce the economic loss, but also for consumer safety (Gerez et al. 2009). In situ antifungal activity of sourdough LAB is related to their antagonistic activity against spoilage molds. This inhibitory effect depends on production of antifungal metabolites such as organic acids, hydroxylated fatty acids, cyclic dipeptides, and antifungal peptides, as well as their interactions. Some mechanisms involved in this antagonistic hurdle are well defined. Destruction of fungi membrane or increasing of membrane permeability and quorum sensing are some of the most important mode of actions revealed for the inhibitory metabolites (Axel et al. 2017). Ryan et al. (2008) reported that in both in vitro and in situ investigations, the sourdough fermented by antifungal Lact. plantarum strains significantly affected the outgrowth of Aspergillus, Fusarium, and Penicillium as common bread spoilage fungi. Furthermore, a clear increase in bread shelf life was achieved when antifungal sourdoughs and calcium propionate were combined. According to these findings, antifungal sourdough can be used in order to reduce the amounts of chemical preservatives in bread making. The sourdough extracts analysis revealed the occurrence of a series of peptides in inhibitory fraction obtained from the antifungal selected LAB isolates in Garofalo et al. (2012) study. Acetic and phenyllactic acids were also identified as the major antifungal metabolites produced by protective $\mathrm{LAB}$ cultures in Gerez et al. (2009) survey. In aforementioned research, selected LAB isolates displayed antifungal activity against the main fungal contaminants in the produced sourdough bread. Zhang et al. (2010) announced that the acetate and propionate (as the key metabolites in inhibiting mold growth) levels produced in sourdough with low, medium, and high ash contents were increased by increasing the buffering capacities of the flours. In the present study, a combination of the selected and non-selected $\mathrm{LAB}$ isolates with total counts of the $8.4 \pm 0.3 \mathrm{Log} \mathrm{CFU} / \mathrm{g}$ was detected in the spontaneous sourdough, whereas in the controlled sourdough the same amount of the selected $\mathrm{LAB}$ was added. It is assumed that except the presence of the selected LAB, there are several other factors (such as its population, the interaction between the selected and non-selected $\mathrm{LAB}$, and probably interactions among their metabolites) influencing the in situ antifungal activity of the selected LAB in spontaneous sourdough unlike the controlled sourdough. The antifungal activity of sourdough LAB in bread is also attributed to synergistic interactions of the LAB metabolites with dough or microbial derived compounds (Lavermicocca et al. 2000).

\section{Conclusion}

In conclusion, Ped. pentosaceus was identified as the selected LAB isolated from wheat sourdough, and then, its in vitro antifungal activity and acidification capacity were 

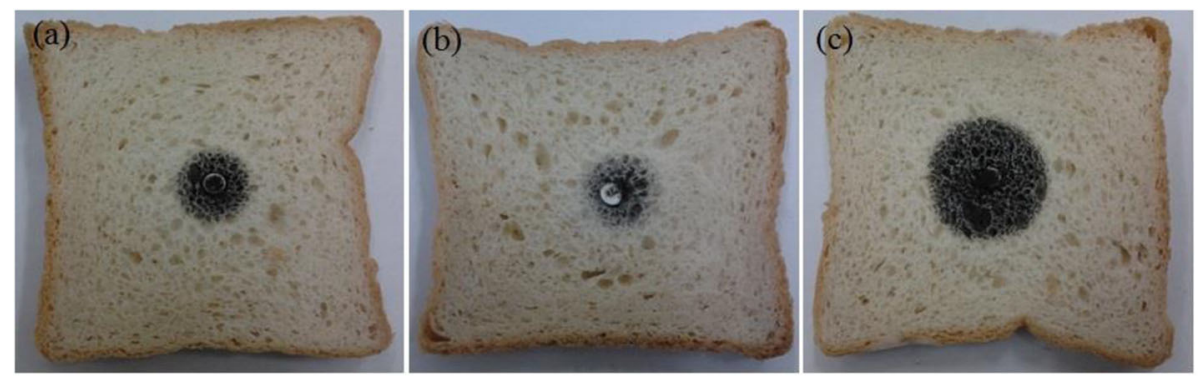

Fig. 5 A. niger growth on bread samples containing spontaneous (without the starter culture) sourdough (a) and controlled (containing the Ped. pentosaceus starter culture) sourdough (b) in comparison with the control (without sourdough) bread (c), 7 days after baking

verified. Active components in antifungal fraction obtained from preparative TLC of the LAB culture filtrate identified using GC/MS were also 3-isobutyl 2,5 piperazinedione, 9,12-octadecadienoic acid 1-methylethyl ester, 9-octadecenoic acid 2-hydroxy-1-(hydroxymethyl) ethyl ester, and pyrrolo[1,2-a]pyrazine-1,4-dione, hexahydro7-hydroxy-phenylmethyl. Furthermore, anti-aflatoxigenic capability of Ped. pentosaceus isolate was verified using HPLC analysis. The hardness, porosity, and OA were measured $5.02 \mathrm{~N}, 23.16 \%$, and 4.06, respectively in bread containing controlled sourdough fermented with Ped. pentosaceus isolate as the starter culture. Moreover, PA content and fungal spoilage of controlled sourdough bread remarkably decreased in comparison with the control bread. In accordance with these results, application of the controlled sourdough containing Ped. pentosaceus isolate in loaf bread is important from technological, sensorial, and functional viewpoints in processing of this product.

\section{Authors' contributions}

The authors read and approved the final manuscript.

\section{Funding}

This study was financially supported by the Iran National Science Foundation (INSF 93051600 Grant), and the authors thank the support.

\section{Ethics approval and consent to participate}

Not applicable for ATHLOS study: ethics approval and participants' consent to participate were obtained by each study at the time point of surveys' field trials.

\section{Competing interests}

The authors declare that they have no conflict of interest.

\section{Author details}

${ }^{1}$ Food, Drug and Natural Products Health Research Center, Golestan University of Medical Sciences, Gorgan, Iran. ${ }^{2}$ Department of Food Science and Technology, Gorgan University of Agricultural Sciences and Natural Resources, Gorgan 49138-15739, Iran. ${ }^{3}$ Department of Food Science and Technology, Ferdowsi University of Mashhad, Mashhad, Iran.

Received: 21 September 2019 Accepted: 23 April 2020

Published online: 04 May 2020

\section{References}

AACC (2010) Moisture 44-19, protein 46-10, gluten 38-12, fat 30-10, carbohydrate 39-21 and ash 08-01 methods. American Association of Cereal Chemists (AACC) International, St. Paul
Abnous K, Brooks SP, Kwan J, Matias F, Green-Johnson J, Selinger LB, Thomas M, Kalmokoff M (2009) Diets enriched in oat bran or wheat bran temporally and differentially alter the composition of the fecal community of rats. J Nut 139: 2024-2031

Alfonzo A, Ventimiglia G, Corona O, Di Gerlando R, Gaglio R, Francesca N, Moschetti G, Settanni L (2013) Diversity and technological potential of lactic acid bacteria of wheat flours. Food Microbiol 36:343-354

Arendt EK, Ryan LA, Dal Bello F (2007) Impact of sourdough on the texture of bread. Food Microbiol 24:165-174

Axel C, Zannini E, Arendt EK (2017) Mold spoilage of bread and its biopreservation: a review of current strategies for bread shelf life extension. Crit Rev Food Sci 57:3528-3542

Carson L, Setser C, Sun XS (2000) Sensory characteristics of sorghum composite bread. Int J Food Sci Technol 35:465-471

Clarke Cl, Arendt EK (2005) A review of the application of sourdough technology to wheat breads. Adv Food Nut Res 49:137-161

Dal Bello F, Clarke C, Ryan L, Ulmer H, Schober T, Ström K, Sjögren J, Van Sinderen D, Schnürer J, Arendt E (2007) Improvement of the quality and shelf life of wheat bread by fermentation with the antifungal strain Lactobacillus plantarum FST 1.7. J Cereal Sci 45:309-318

Dalié D, Deschamps A, Richard-Forget F (2010) Lactic acid bacteria-potential for control of mould growth and mycotoxins: a review. Food Control 21:370-380

De Vuyst L, Van Kerrebroeck S, Harth H, Huys G, Daniel HM, Weckx S (2014) Microbial ecology of sourdough fermentations: diverse or uniform? Food Microbiol 37:11-29

El-Nezami H, Kankaanpää PE, Salminen S, Ahokas J (1998) Physicochemical alterations enhance the ability of dairy strains of lactic acid bacteria to remove aflatoxin from contaminated media. J Food Protect 61:466-468

Garofalo C, Zannini E, Aquilanti L, Silvestri G, Fierro O, Picariello G, Clementi F (2012) Selection of sourdough lactobacilli with antifungal activity for use as biopreservatives in bakery products. J Agric Food Chem 60:7719-7728

Gerez CL, Torino Ml, Rollán G, De Valdez GF (2009) Prevention of bread mould spoilage by using lactic acid bacteria with antifungal properties. Food Control 20:144-148

Gerez CL, Torres MJ, De Valdez GF, Rollán G (2013) Control of spoilage fungi by lactic acid bacteria. Biol Control 64:231-237

Gobbetti M (1998) The sourdough microflora: interactions of lactic acid bacteria and yeasts. Trends Food Sci Tech 9:267-274

Gobbetti M, Rizzello CG, Di Cagno R, De Angelis M (2014) How the sourdough may affect the functional features of leavened baked goods. Food Microbiol 37:30-40

Guimarães A, Santiago A, Teixeira JA, Venâncio A, Abrunhosa L (2018) Antiaflatoxigenic effect of organic acids produced by Lactobacillus plantarum. Int J Food Microbiol 264:31-38

Haskard CA, El-Nezami HS, Kankaanpää PE, Salminen S, Ahokas JT (2001) Surface binding of aflatoxin B1 by lactic acid bacteria. Appl Environ Microbiol 67: 3086-3091

Hassan YI, Bullerman LB (2008) Antifungal activity of Lactobacillus paracasei subsp. tolerans against Fusarium proliferatum and Fusarium graminearum in a liquid culture setting. J Food Prot 71:2213-2216

Haug W, Lantzsch HJ (1983) Sensitive method for the rapid determination of phytate in cereals and cereal products. J Sci Food Agr 34:1423-1426 
Katina K, Heiniö RL, Autio K, Poutanen K (2006) Optimization of sourdough process for improved sensory profile and texture of wheat bread. LWT-Food Sci Technol 39:1189-1202

Lavermicocca P, Valerio F, Evidente A, Lazzaroni S, Corsetti A, Gobbetti M (2000) Purification and characterization of novel antifungal compounds from the sourdough Lactobacillus plantarum strain 21B. Appl Environ Microbiol 66: 4084-4090

Le Lay C, Mounier J, Vasseur V, Weill A, Le Blay G, Barbier G, Coton E (2016) In vitro and in situ screening of lactic acid bacteria and propionibacteria antifungal activities against bakery product spoilage molds. Food Control 60: 247-255

Lee HM, Lee $Y$ (2008) A differential medium for lactic acid-producing bacteria in a mixed culture. Lett Appl Microbiol 46:676-681

Leenhardt F, Levrat-Verny MA, Chanliaud E, Rémésy C (2005) Moderate decrease of $\mathrm{pH}$ by sourdough fermentation is sufficient to reduce phytate content of whole wheat flour through endogenous phytase activity. J Agr Food Chem 53:98-102

Lopez HW, Duclos V, Coudray C, Krespine V, Feillet-Coudray C, Messager A, Demigné C, Rémésy C (2003) Making bread with sourdough improves mineral bioavailability from reconstituted whole wheat flour in rats. Nutrition 19:524-530

Magnusson J, Schnürer J (2001) Lactobacillus coryniformis subsp. coryniformis strain Si3 produces a broad-spectrum proteinaceous antifungal compound. Appl Environ Microbiol 67:1-5

Manini F, Casiraghi M, Poutanen K, Brasca M, Erba D, Plumed-Ferrer C (2016) Characterization of lactic acid bacteria isolated from wheat bran sourdough. LWT-Food Sci Technol 66:275-283

Niderkorn V, Morgavi D, Aboab B, Lemaire M, Boudra H (2009) Cell wall component and mycotoxin moieties involved in the binding of fumonisin B1 and B2 by lactic acid bacteria. J Appl Microbiol 106:977-985

Palacios MC, Haros M, Sanz Y, Rosell CM (2008) Selection of lactic acid bacteria with high phytate degrading activity for application in whole wheat breadmaking. LWT-Food Sci Technol 41:82-92

Rizzello CG, Nionelli L, Coda R, Di Cagno R, Gobbetti M (2010) Use of sourdough fermented wheat germ for enhancing the nutritional, texture and sensory characteristics of the white bread. Eur Food Res Technol 230:645-654

Russo P, Arena MP, Fiocco D, Capozzi V, Drider D, Spano G (2017) Lactobacillus plantarum with broad antifungal activity: a promising approach to increase safety and shelf-life of cereal-based products. Int J Food Microbiol 247:48-54

Ryan LAM, Dal Bello F, Arendt EK (2008) The use of sourdough fermented by antifungal $L A B$ to reduce the amount of calcium propionate in bread. Int J Food Microbiol 125:274-278

Saladino F, Luz C, Manyes L, Fernández-Franzón M, Meca G (2016) In vitro antifungal activity of lactic acid bacteria against mycotoxigenic fungi and their application in loaf bread shelf life improvement. Food Control 67:273277

Sellamani M, Kalagatur NK, Siddaiah C, Mudili V, Krishna K, Natarajan G, Putcha R, Venkata $L$ (2016) Antifungal and zearalenone inhibitory activity of Pediococcus pentosaceus isolated from dairy products on Fusarium graminearum. Front Microbiol 7:890

Ström K, Sjögren J, Broberg A, Schnürer J (2002) Lactobacillus plantarum MiLAB 393 produces the antifungal cyclic dipeptides cyclo (L-Phe-L-Pro) and cyclo (L-Phe-trans-4-OH-L-Pro) and 3-phenyllactic acid. Appl Environ Microbiol 68 $4322-4327$

Tamura K, Stecher G, Peterson D, Filipski A, Kumar S (2013) MEGA6: molecular evolutionary genetics analysis version 6.0. Mol Biol Evol 30:2725-2729

Yan PS, Song Y, Sakuno E, Nakajima H, Nakagawa H, Yabe K (2004) Cyclo (Lleucyl-L-prolyl) produced by Achromobacter xylosoxidans inhibits aflatoxin production by Aspergillus parasiticus. Appl Environ Microbiol 70:7466-7473

Zhang C, Brandt MJ, Schwab C, Gänzle MG (2010) Propionic acid production by cofermentation of Lactobacillus buchneri and Lactobacillus diolivorans in sourdough. Food Microbiol 27:390-395

Zwietering M, Jongenburger I, Rombouts F, Van't Riet K (1990) Modeling of the bacterial growth curve. Appl Environ Microbiol 56:1875-1881

\section{Publisher's Note}

Springer Nature remains neutral with regard to jurisdictional claims in published maps and institutional affiliations.

\section{Ready to submit your research? Choose BMC and benefit from:}

- fast, convenient online submission

- thorough peer review by experienced researchers in your field

- rapid publication on acceptance

- support for research data, including large and complex data types

- gold Open Access which fosters wider collaboration and increased citations

- maximum visibility for your research: over $100 \mathrm{M}$ website views per year

At $\mathrm{BMC}$, research is always in progress.

Learn more biomedcentral.com/submissions 